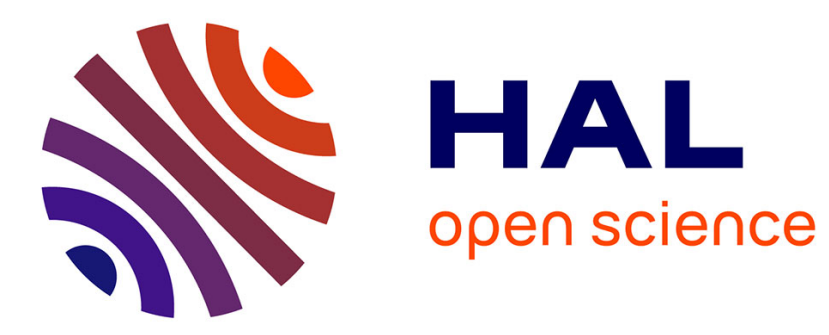

\title{
Disentangling theorem and scattering functions for long-range coherent tunneling
}

Peter S M Townsend, Alex W Chin

\section{To cite this version:}

Peter S M Townsend, Alex W Chin. Disentangling theorem and scattering functions for long-range coherent tunneling. Physical Review A, 2019, 99 (1), 10.1103/PhysRevA.99.012112 . hal-02377565

\section{HAL Id: hal-02377565 https://hal.sorbonne-universite.fr/hal-02377565}

Submitted on 23 Nov 2019

HAL is a multi-disciplinary open access archive for the deposit and dissemination of scientific research documents, whether they are published or not. The documents may come from teaching and research institutions in France or abroad, or from public or private research centers.
L'archive ouverte pluridisciplinaire HAL, est destinée au dépôt et à la diffusion de documents scientifiques de niveau recherche, publiés ou non, émanant des établissements d'enseignement et de recherche français ou étrangers, des laboratoires publics ou privés. 


\title{
Disentangling theorem and scattering functions for long-range coherent tunneling
}

\author{
Peter S. M. Townsend* \\ Surfaces, Microstructure and Fracture Group, Department of Physics, Cavendish Laboratory, \\ J.J. Thomson Avenue, Cambridge CB3OHE, United Kingdom
}

Alex W. Chin

Centre National de la Recherche Scientifique, Institute des Nanosciences de Paris, Sorbonne Université, 4 place Jussieu, 75005 Paris, France

(Received 23 October 2018; published 14 January 2019)

\begin{abstract}
A closed form of the disentangling theorem is used to derive an exact expression for the quantum mechanical intermediate scattering function describing long-range coherent quantum tunneling. The result applies to a single particle in a large periodic nearest-neighbor tight-binding system in one spatial dimension, with one localized site per unit cell. The result is exact up to the assumption of orthogonal localized states and the substitution of the coordinate operator with a discrete representation diagonal in the on-site basis. The intermediate scattering function is expressed in terms of modified Bessel functions, and consists of a symmetric real part and antisymmetric imaginary part. The real and imaginary parts both exhibit decaying oscillations reflecting the oscillatory dynamics among neighboring sites combined with the long-term spreading of the wave function from any initial site. The imaginary part is significant only when the thermal energy is comparable to or smaller than the width of the tight-binding energy band, and represents quantum recoil or the asymmetry of energy exchange probability in quasielastic scattering from the coherent system. The one-dimensional result is extended, in the form of k-space integrals, to describe the coherent tunneling dynamics in hexagonal and honeycomb systems. The prospects for observing the phenomenology of the analytical line shapes are discussed with respect to the practical implementation of helium-3 surface spin echo spectroscopy.
\end{abstract}

DOI: 10.1103/PhysRevA.99.012112

\section{INTRODUCTION}

The phenomenon of quantum tunneling, the passage of a quantum system through a configuration forbidden by classical mechanics, is relevant to a huge range of physical processes. Examples include alpha decay [1], nuclear fusion [2], intermolecular hydrogen transfer [3], bimolecular chemical reactions [4], the dynamics of superconducting junctions [5], surface diffusion [6], and the postulated future decay of the entire Universe [7]. Tunneling is a clear manifestation of completely nonclassical behavior in physical systems and acts as an important conceptual reference point between the diverse mathematical approaches to quantum mechanics. For example, tunneling is describable by delocalized stationary states in wave mechanics [8], or instantons and bounces in the path integral formalism [9-11]. Alternatively, a very efficient description of tunneling can be achieved by a finite basis matrix mechanics representation in which the sole dynamics of the system are those induced by tunneling. In any formalism, the extent of quantum coherence in tunneling in the presence of a dissipative environment is a key issue when trying to describe real systems, and coherent tunneling is highly relevant to fields such as quantum computing [12], quantum biology $[13,14]$, and transport in organic semiconductors [15]. In the

*peter.townsend@rutgers.edu; present address: Rutgers University, Department of Chemistry and Chemical Biology, 123 Bevier Road, Piscataway, NJ 08854, USA. present work a recently derived form of the Baker-Hausdorff disentangling theorem for exponentiated operators is used to derive an exact scattering function for coherent tunneling in a periodic system described by a single tight-binding band. The result is relevant to experimental investigations of quantum diffusion using spectroscopic, quasielastic scattering methods.

Experimental surface dynamics measurements can be broadly divided into real-space techniques such as microscopies, and scattering measurements providing information in reciprocal space. Scanning tunneling microscopy has been widely applied to study quantum diffusion at surfaces $[6,16,17]$, but the relatively long accessible timescales and the invasive effect of the tip limit the prospects for observing coherent dynamics. By contrast, quasielastic scattering techniques provide full access to coherent dynamics of diffusing species, for example, via the intermediate scattering function (ISF) which is accessible in Fourier transform methods based on the spin-echo principle [18-20]. Quasielastic neutron scattering has previously been used to observe partially coherent two-state tunneling dynamics of hydrogen atoms near bulk impurities [21] and to investigate the associated predictions of two-state dissipative tunneling theory [22] in a metallic environment. The depth of physical information available from such measurements motivates the further development of theoretical line shapes for scattering experiments capable of resolving coherent tunneling dynamics. In surface diffusion on close-packed metal substrates, appreciable tunneling amplitudes are expected for atomic tunneling between equivalent adsorption sites that form an extended periodic system 


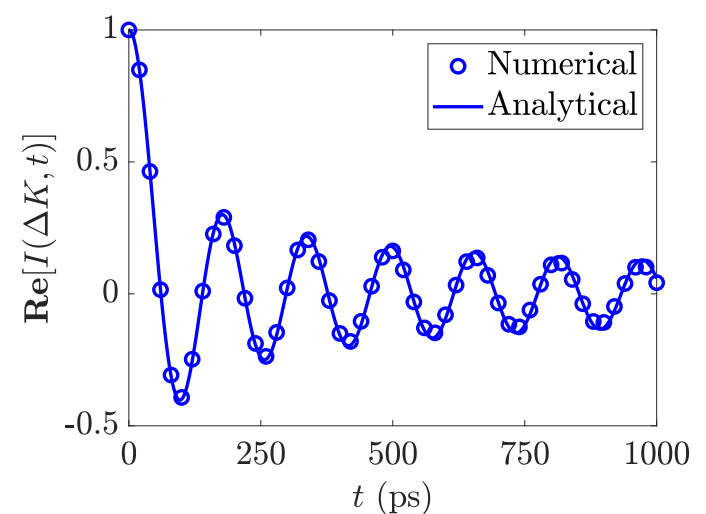

(a)

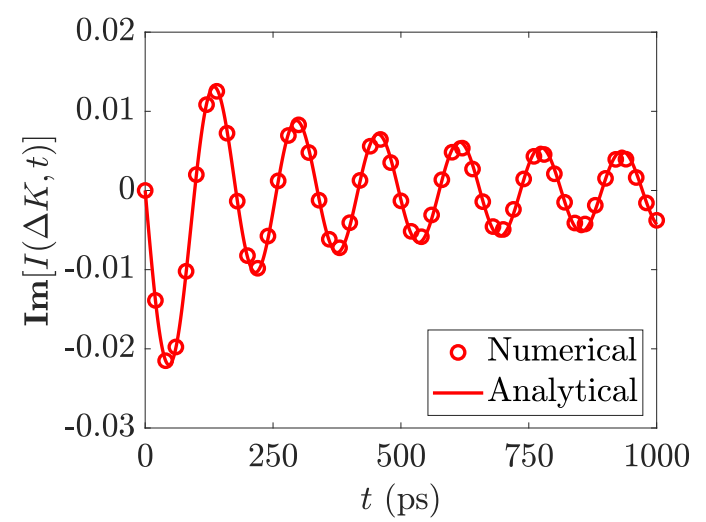

(b)

FIG. 1. Illustration of the ISF (31) describing coherent tunneling in a tight-binding system, with parameters $\delta=0.01 \mathrm{meV}, \Delta K=$ $1.8 \AA^{-1}, \mathrm{a}=2.7 \AA, \mathrm{T}=4.0 \mathrm{~K}$. The analytical formula is represented by solid curves. (a) The real part is shown in the upper plot; (b) the imaginary part in the lower plot. The real (imaginary) part is symmetric (antisymmetric) with respect to the correlation time $t$. Numerical results obtained by evaluating the expression (10) in a large finite basis are shown as open circles. The imaginary part becomes insignificant when $k_{B} T \gg \hbar \delta$.

$[23,24]$ rather than a two-state system. Therefore, in principle, motion linked to long-range coherent tunneling could be observed in surface-specific experiments under appropriate conditions. High-resolution measurement of reciprocal-space surface dynamics is possible using helium-3 surface spin-echo spectroscopy (HeSE) [20], and the interpretation of HeSE data generally relies on models for the ISF. Scientific progress associated with the technique is not driven simply by an accumulation of systems measured, but by the development of analytical models for potentially observable surface dynamical processes [25-30] in parallel with ongoing improvements in instrumentation [31-35], data acquisition routines [36,37], and analysis methods [38,39]. The main analytical result and method presented here provides a new class of contribution in that context. The extent to which observation of the analytical phenomenology may be observable with current instrumentation will be discussed further in Sec. III.

In the present work, coherent tunneling in a large periodic system of $N$ equivalent sites is modeled using a tight-binding Hamiltonian. In the tight-binding model, a restricted basis set of one localized state per unit cell is assumed, and the localized states are coupled with a parameter that controls the mobility of the particle, or the bandwidth. The tightbinding concept may be familiar as a model for electronic band structure evaluation [40,41], but can equally well be used as a generic model for tunneling in a multisite system [42-44]. In the special case $N=2$, the tight-binding model describes a two-state system, for which the dynamical structure factor is already known [45,46], and describes confined coherent oscillations between neighboring sites with no longterm spreading of the wave function. The derivation of the dynamical structure factor in the two-state example is facilitated by convenient mathematical properties of Pauli matrices. By contrast the calculation for large $N$, which describes the interplay of local coherent oscillations combined with the long-term spreading of the wave function in coherent quantum diffusion, can be conveniently carried out using results from a different branch of quantum operator theory, namely the Baker-Campbell-Hausdorff (BCH) distentangling theorem [47-49].

The disentangling theorem, an infinite-series expression for $\ln \left(e^{X} e^{Y}\right)$ for noncommuting operators $X$ and $Y$, is a common feature in previous analytical derivations of quantum intermediate scattering functions [50-52]. The disentangling operation for canonical operators whose commutator $[X, Y]$ is a complex number leads to the appearance of an antisymmetric imaginary part in the correlation function. The imaginary part is required by the detailed balance constraint in quasielastic scattering from a thermal system [50], and can also be interpreted in terms of the recoil of the scattering centres to conserve momentum when imparting a momentum transfer to a scattering probe. The imaginary part vanishes if $\hbar \rightarrow 0$, and is therefore referred to as quantum recoil [51]. Although the closed form of the disentangling theorem for complex-number commutators $[X, Y]$ is a standard and widely known result [53], further closed formulas and algorithms for evaluating the $\mathrm{BCH}$ series are still being actively developed and classified in the framework of group theory [54-57]. In Sec. II, a recent closed-form version of the disentangling theorem [54], which holds under a more general class of commutation relations, is used to derive the exact real and imaginary parts of the intermediate scattering function for a particle in a tight-binding system. In Sec. III the potential generalization of the result is discussed along with prospects for observing the coherent phenomenology in surface diffusion experiments. The conclusions are summarized in Sec. VI.

\section{QUANTUM MECHANICAL SCATTERING FUNCTION}

For a particle with position operator $x$ in a quantum system with one spatial dimension, governed by a time-independent Hamiltonian $H$, the quantum ISF is defined in terms of the Heisenberg representation of time evolution [49]. A general operator $A$ evolves according to

$$
A(t)=\exp \left(\frac{i H t}{\hbar}\right) A(0) \exp \left(\frac{-i H t}{\hbar}\right) .
$$

The ISF is a function of a correlation time $t$ and a momentum transfer $\Delta K$. Additionally, as an equilibrium correlation function the ISF depends on the temperature $T$ of the system, 
more conveniently expressed via the inverse temperature $\beta=$ $\left(k_{B} T\right)^{-1}$. Using $x(t)$ to represent the time evolution of $x$ the particle position operator, the quantum ISF can then be written as [51]

$$
I(\Delta K, t)=\frac{\operatorname{tr}\left\{e^{i \Delta K x(t)} e^{-i \Delta K x(0)} e^{-\beta H}\right\}}{\operatorname{tr}\left\{e^{-\beta H}\right\}} .
$$

The form (2) defines the ISF is the Fourier transform to the time domain of the dynamical structure factor $S(\Delta K, \omega)$ $[50,58]$, which is expressed in terms of the global energy eigenstates $\{|k\rangle\}$ and $\left\{\left|k^{\prime}\right\rangle\right\}$ of energies $\left\{\hbar \omega_{k}\right\}$ and $\left\{\hbar \omega_{k^{\prime}}\right\}$ :

$$
S(\Delta K, \omega) \propto \sum_{k, k^{\prime}} e^{-\beta \hbar \omega_{k^{\prime}}}\left|\left\langle k\left|e^{i \Delta K x}\right| k^{\prime}\right\rangle\right|^{2} \delta\left(\omega-\left[\omega_{k}-\omega_{k^{\prime}}\right]\right),
$$

where $\delta(\omega)$ represents a Dirac delta function. The normalization in the definition (2) ensures that $I(\Delta K, 0)=1$. When the number of accessible energy eigenstates of the system is finite, elastic contributions $\left(\omega_{k}=\omega_{k^{\prime}}\right)$ will typically represent a finite fraction of $S(\Delta K, \omega)$ whereas for large $N$ it is possible for the elastic contribution to vanish, which will be a major difference between the $N=2$ two-state system result and the large- $N$ result for long-range tunneling.

Here the ISF is computed from the definition (2) for coherent tunneling in an extended $N$-site tight-binding system with one accessible energy band [40,41]. Let $\{|n\rangle\}$ be the localized states of the system associated with the accessible band, that is, the Wannier states of the band. We will assume that the Wannier states are mutually orthogonal, which is a good approximation for strongly localized states. Let $\delta$ represent the matrix element $\langle n|H| n+1\rangle$ of the Hamiltonian operator $H$ between neighboring Wannier states. We will assume for simplicity that $\delta$ is real, and that the Hamiltonian matrix elements coupling more distant neighbors are all zero. Let $\phi$ and $\chi$ represent the auxiliary operators $\phi$ and $\chi$, finite translation operators in the positive and negative directions, respectively,

$$
\phi=\sum_{n}|n+1\rangle\left\langle n\left|; \quad \chi=\sum_{n}\right| n-1\right\rangle\langle n| .
$$

Then, the model Hamiltonian reads

$$
H=(\phi+\chi) \delta .
$$

It is convenient to define also the discrete position operator $n$ whose eigenvalues are the indices of the localized sites in the system

$$
n=\sum_{n^{\prime}} n^{\prime}\left|n^{\prime}\right\rangle\left\langle n^{\prime}\right| .
$$

The commutation relations between $n, \chi$, and $\phi$, which generate the time evolution of $n$, then follow as

$$
[\chi, \phi]=0, \quad[\chi, n]=\chi, \quad[\phi, n]=-\phi .
$$

Assuming periodic boundary conditions site $N+1$ is equivalent to site 1 and the eigenstates of $H$ are Bloch states $[40,41]$ of the form

$$
|k\rangle=\frac{1}{\sqrt{N}} \sum_{n} e^{i k n}|n\rangle(0 \leqslant k<2 \pi),
$$

where $k$ is a dimensionless crystal momentum. Here $N$ will be assumed large enough for the allowed values of $k$ to be treated as a continuum when performing sums in $k$ space. The energy of the Bloch states is

$$
\epsilon(k)=-2 \delta \cos (k) .
$$

Following the treatment of the two-state system briefly reviewed above [45], the ISF for the tight-binding system can be approximated by replacing $x$, the position operator with a continuous spectrum, with na where $a$ is the lattice parameter and $n$ is the discrete position operator (6). The quantum ISF (2) then becomes

$$
I(\Delta K, t)=\operatorname{tr}\left\{e^{i \Delta K a n(t)} e^{-i \Delta K a n(0)} e^{-\beta H}\right\} / \operatorname{tr}\left\{e^{-\beta H}\right\} .
$$

The time evolution of $n(t)$ follows straightforwardly from the standard operator equation of motion $\dot{n}=i[H, n] / \hbar$. From the commutation relations (7) the closed-form evolution of $n(t)$ is given in terms of the Schrödinger operator $n \equiv n(0)$ by

$$
n(t)=n+\frac{i \delta}{\hbar}(\chi-\phi) t .
$$

After substituting $n(t)$ into the definition of $I(\Delta K, t)$, the derivation proceeds very much like the free-particle ballistic case [51] by applying the disentangling theorem. We use a special case [54] of the $\mathrm{BCH}$ disentangling theorem. The special case applies when the commutator of two operators $X$ and $Y$ is a linear combination of $X, Y$ and the identity operator $\mathcal{I}$ :

$$
[X, Y]=u X+v Y+c \mathcal{I},
$$

where $u, v$, and $c$ are complex numbers. Under such conditions the product of the exponentiated operators is

$$
e^{X} e^{Y}=\exp (X+Y+f(u, v)[X, Y]),
$$

with $f(u, v)$ given by

$$
f(u, v)=\frac{(u-v) e^{u+v}-\left(u e^{u}-v e^{v}\right)}{u v\left(e^{u}-e^{v}\right)} .
$$

In the present application, the commutation relation can be specialized to the form

$$
[X, Y]=u X,
$$

and so the result can be simplified further by taking the limit $v \rightarrow 0$, giving

$$
e^{X} e^{Y}=\exp [X+Y+u f(u) X],
$$

where the scalar function $f(u)=f(u, v \rightarrow 0)$ is given by

$$
f(u)=\frac{1+e^{u}(u-1)}{u\left(e^{u}-1\right)} .
$$

The corresponding Zassenhaus formula $[59,60]$ for $\exp (X+$ $Y$ ) can be derived by inverting Eq. (16), and reads

$$
\exp (X+Y)=\exp \left[\frac{X}{1+u f(u)}\right] \exp (Y) .
$$

Equation (18) can reduce the operator appearing inside the trace of Eq. (10) to a form involving only $\phi$ and $\chi$, independent of $n$, which allows straightforward evaluation of 
$I(\Delta K, t)$ in the Bloch basis, by writing out the time-evolved exponential operator

$$
e^{i \Delta K a n(t)}=\exp \left[i \Delta K a n-\Delta K a \frac{t \delta}{\hbar} \chi+\Delta K a \frac{t \delta}{\hbar} \phi\right],
$$

and making the substitutions

$$
X=\Delta K a \frac{t \delta}{\hbar} \phi, \quad Y=i \Delta K a n-\Delta K a \frac{t \delta}{\hbar} \chi .
$$

Applying the disentangling result (18) turns the right-hand side of Eq. (19) into

$$
\exp \left[\frac{\Delta K a t \delta \phi / \hbar}{1-i \Delta K a f(-i \Delta K a)}\right] \exp \left[i \Delta K a n-\Delta K a \frac{t \delta}{\hbar} \chi\right] \text {. }
$$

Further applying the disentangling Eq. (18) to Eq. (21) with the new substitutions

$$
X=-\Delta K a \frac{t \delta}{\hbar} \chi, \quad Y=i \Delta K a n,
$$

gives the fully factorized version of $\exp [i \Delta K a n(t)]$. Introducing the function

$$
g(u)=\frac{1}{1+u f(u)},
$$

the result can be written as

$$
\begin{aligned}
e^{i \Delta K a n(t)}= & \exp \left\{\Delta K \frac{t \delta}{\hbar} a[g(-i \Delta K a) \phi-g(i \Delta K a) \chi]\right\} \\
& \times e^{i \Delta K a n(0)}
\end{aligned}
$$

The third and final exponential factor $e^{i \Delta K a n}$, a function of the Schrödinger operator $n$, cancels within the ISF definition (2), leaving us with

$$
I(\Delta K, t)=\frac{\operatorname{tr}\left\{\exp \left(\Delta K \frac{t \delta}{\hbar} a[g(-i \Delta K a) \phi-g(i \Delta K a) \chi]\right) e^{-\beta H}\right\}}{\operatorname{tr}\left\{e^{-\beta H}\right\}} .
$$

Since $\phi|k\rangle=e^{-i k}|k\rangle$ and $\chi|k\rangle=e^{i k}|k\rangle$, the trace is conveniently evaluated in the $|k\rangle$ basis. Assuming a large number of sites $N$ giving a continuum of $k$ states, the ISF numerator is given by

$$
\frac{1}{2 \pi} \int_{0}^{2 \pi} d k e^{-2 \beta \delta \cos (k)} \exp \left\{-\frac{t \delta}{\hbar} a \Delta K\left[g(i \Delta K a) e^{i k}-g(-i \Delta K a) e^{-i k}\right]\right\} .
$$

$g(i \Delta K a)$ and $g(-i \Delta K a)$ are complex conjugates, and so by breaking $g(i \Delta K a)$ up into real and imaginary parts $g^{\prime}$ and $g^{\prime \prime}$, respectively, according to

$$
g(i \Delta K a)=g^{\prime}+i g^{\prime \prime},
$$

the integral can be recast as

$$
\frac{1}{2 \pi} \int_{0}^{2 \pi} d k \exp [A \cos (k)+B \sin (k)]
$$

where

$$
A=-2 \beta \delta-2 i \frac{t \delta}{\hbar} \Delta K a g^{\prime \prime}
$$

and

$$
B=-2 i \frac{t \delta}{\hbar} \Delta K a g^{\prime}
$$

The integral (28) has a standard special-function representation in terms of the zeroth-order modified Bessel function of the first kind $I_{0}(x)$ [61]. The correctly normalized ISF is given by

$$
I(\Delta K, t)=\frac{I_{0}\left(\sqrt{A^{2}+B^{2}}\right)}{I_{0}(2 \beta \delta)} .
$$

Equation (31), together with the relations (29) and (30) and the definition of the function $g(i \Delta K a)$ and its real and imaginary parts, constitutes the central analytical result of the current study.
Figure 1 illustrates the analytical result (31) for the parameter set $\delta=0.01 \mathrm{meV}, \Delta K=1.8 \AA, \mathrm{a}=2.7 \AA, \mathrm{T}=4.0 \mathrm{~K}$. The upper plot shows the real part, which is symmetric with respect to the correlation time, and the lower plot shows the imaginary part, which is antisymmetric. In both plots, solid lines indicate the analytical result. For comparison, numerical samples obtained by direct matrix evaluation of Eq. (10) in a finite basis with $N=250$ are shown as open circles. Correlation times up to $1 \mathrm{~ns}$ are included, consistent with the range of correlation time currently accessible using present helium-3 surface spin echo spectroscopy instrumentation $[20,39,62,63]$.

\section{DISCUSSION}

\section{A. Relation to $S(\Delta K, \omega)$ and recoil}

The main result of the present work is represented by Eq. (31) and Fig. 1. The result holds for a periodic onedimensional tight-binding system with one localized state per unit cell, a large number of unit cells, and a discrete approximation for the position operator. The quantum ISF consists of an oscillatory decaying symmetric real part, and an oscillatory decaying antisymmetric imaginary part that becomes negligible when $k_{B} T \gg \hbar \delta$. An alternative perspective on the imaginary part, which draws a close connection to quantum recoil in continuous systems [30,51,52], can be seen by casting the integral representation (28) in terms of shifted energy and crystal momentum. Namely, by using standard double-angle trigonometric identities and the explicit forms 


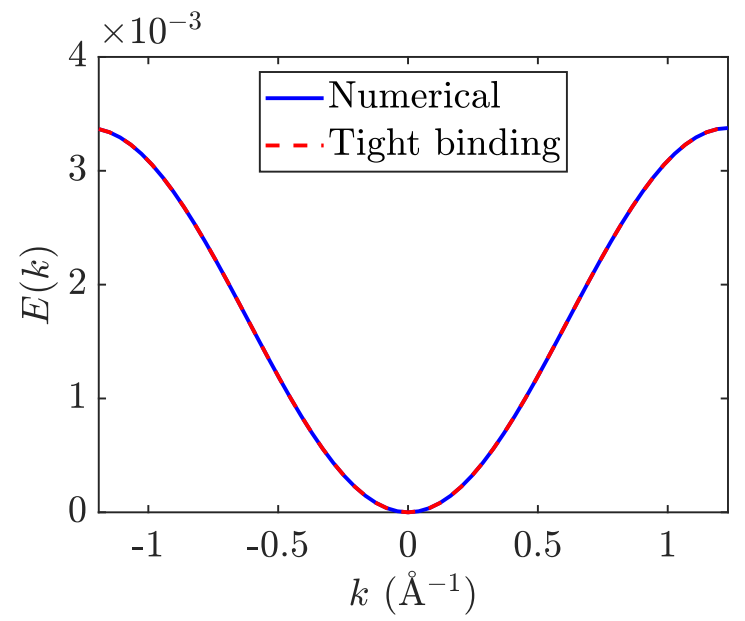

FIG. 2. The lowest-energy band for a particle in a continuous cosine potential as described by the Hamiltonian (33) with parameters $V_{0}=50 \mathrm{meV}$ (barrier height $100 \mathrm{meV}$ ), and periodicity $a=2.55 \AA$. The blue solid curve gives the numerically exact dispersion relation $E(k)$ as a function of the crystal momentum. The red dashed line is the tight-binding dispersion relation of Eq. (9) where the tunnel matrix element $\delta$ is chosen to match the bandwidth of the numerical result.

of $g^{\prime}$ and $g^{\prime \prime}$, one finds an integral of the form

$$
\frac{1}{2 \pi} \int_{0}^{2 \pi} d k e^{-\beta \hbar \omega(k)} \exp \{i t[\omega(k)-\omega(k-\Delta K a)]\},
$$

where $\hbar \omega(k)$ is the energy dispersion (9). The appearance of the shifted $\omega(k-\Delta K a)$ can be traced back to the conservation of crystal momentum during scattering from the coherent system, in analogy to the energy shift in quantum recoil from a free, ballistic quantum particle [51].

\section{B. Application to cosine potential}

We now analyze the case of coherent tunneling in a continuous cosine potential, using realistic parameters describing surface diffusion of hydrogen, to confirm that the tightbinding model provides a good description of the band structure, and to quantify the effects of dissipation by comparison to an analytical formula for the incoherent tunneling rate.

We write the Hamiltonian of our continuous system for a particle of mass $m$ in a cosine potential of periodicity $a$, as

$$
H=\frac{p^{2}}{2 m}+V_{0} \cos \left(\frac{2 \pi x}{a}\right) .
$$

To compute the band structure, we choose several values of the crystal momentum $k$ inside the first Brillouin zone. The Hamiltonian (33) is known in advance to be block-diagonal in the basis of crystal momentum eigenstates, which allows an efficient $k$-wise computation of the band structure [23,24]. For each $k$ we construct the Hamiltonian matrix in the Fourier (momentum) basis with eigenvalues $\hbar(k \pm n G)$, for $-F \leqslant$ $n \leqslant F$ where $F$ the number of Fourier basis states is increased to convergence, and $G$ is the reciprocal lattice vector $2 \pi / a$. The matrix is diagonalized numerically for each value of $k$.

Figure 2 shows the numerically exact band structure for the parameters $a=2.55 \AA, V_{0}=50 \mathrm{meV}$ (barrier height
$100 \mathrm{meV}), m=1.0 \mathrm{amu}$, using $2 F+1=41$ Fourier basis states per $k$ value. The numerically converged result is shown as a solid line. The dashed line shows the band structure according to the tight-binding model (9) with the parameter $\delta$ fitted from the bandwidth of the numerical results. The tight-binding model provides a good description of the band structure.

Coupling to a dissipative environment has the potential to qualitatively change the nature of the dynamics and the functional form of the ISF. In particular, dissipation generally results in incoherent hopping rather than coherent tunneling. In a one-dimensional system, neglecting quantum recoil effects, and assuming nearest-neighbor hopping with a rate $r$, the ISF associated with ideal Poissonian hopping transport of a single particle is given by [64]

$$
I(\Delta K, t)=\exp (-\alpha(\Delta K)|t|)
$$

where

$$
\alpha(\Delta K)=4 r \sin ^{2}\left(\frac{\Delta K a}{2}\right) .
$$

The $\Delta K$-dependent decay rate $\alpha(\Delta K)$ is somewhat reminiscent of the tight-binding band structure, but the functional form of the ISF (monotonic decay) reflects the incoherent dynamics. We use an analytical formula for the hopping rate to assess the effect of dissipation on the overall decay rate of the ISF in the presence of Langevin friction. The temperature-dependent hopping rate $r(T)$ in a cosine potential can be computed using instanton methods [65] in terms of a dimensionless friction strength $K$

$$
K=\frac{m \gamma a^{2}}{2 \pi \hbar} .
$$

As long as $K \gg 1$, the rate can be evaluated in closed form as

$$
r(T)=\gamma \frac{K \Gamma(1+K) e^{-2 K}}{2 \Gamma\left(\frac{1}{2}\right) \Gamma\left(\frac{1}{2}+K\right)} \frac{\hbar \gamma}{\pi V_{0}}\left(\frac{K k_{B} T}{V_{0}}\right)^{2} .
$$

At low temperatures and at moderate values of the friction $\gamma$, suppression of tunneling by the dissipative coupling to the environment leads to the timescale of incoherent tunneling being much slower than for incoherent tunneling. To quantify the wide disparity in time scales, we evaluate the incoherent rate (37) in a cosine potential of amplitude $V_{0}=50 \mathrm{meV}$, at $T=4 \mathrm{~K}$ with dissipation rate $\gamma=10 \mathrm{ps}^{-1}$. The numerical result is $r(T)=2 \times 10^{-7} \mathrm{ps}^{-1}$. At a scattering condition of $\Delta K=1.0 \AA^{-1}$, in a one-dimensional system with site separation $a=2.55 \AA-1$, the exponential decay rate of the ISF as given by Eq. (35) is larger but of the same order, namely $6.5 \times 10^{-7} \mathrm{ps}^{-1}$. Meanwhile, we can quantify a timescale for the coherent ISF by the correlation time at which the real part of $I(\Delta K, t)$ goes through zero. $t_{0}$ can be estimated by inspection of a plot of the coherent result, but with tightbinding parameter $\delta=$ as derived from the corresponding cosine potential. One finds the first zero at $t_{0} \approx 250 \mathrm{ps}$. So under the conditions considered, the timescale associated with coherent motion is orders of magnitude faster than the timescale for incoherent hopping.

At lower values of the friction, but still sufficiently strong to impose rate dynamics rather than coherent transport, the 
incoherent hopping rate can be computed by direct calculation of dynamical flux correlation functions [66], but the detailed comparison of the low-friction and zero-friction results for realistic surface diffusion potentials is left as further work.

\section{EXTENSIONS}

There are several directions in which the central result can be usefully developed, staying within the framework of completely coherent diffusion. The assumptions behind the result (31) are rather specialized, and so we consider two generalizations of the minimal model (5) of relevance to real surface systems. First, we extend the result to nonseparable two-dimensional motion, exemplified by a hexagonal surface. Second, we extend the result to a system with two degenerate but symmetrically inequivalent sites per unit cell, as exemplified by a honeycomb system, i.e., a graphene-like system [67] or the pattern formed by the threefold hollow sites of fcc(111) and hcp(0001) surfaces.

In the first extension, we derive an expression very similar in form to the integral representation of the one-dimensional result. However, due to the nonseparability of the integration, the final result cannot be expressed in a completely closed form. In the second extension, the result must take into account two bands due to two basis sites per unit cell, and the dynamical structure factor therefore possesses both interband and intraband terms. Again, the result cannot be evaluated in a final closed form. Nevertheless the honeycomb system is an important special case since motion between threefold sites on fcc(111) and hcp(0001) surfaces provide the leading examples of quantum tunneling effects associated with surface diffusion measured by spectroscopic means $[24,62,68]$.

\section{A. Hexagonal lattices}

To develop the two-dimensional result it is convenient to work by generalizing the formula (32). Consider a Hamiltonian for a two-dimensional periodic tight-binding system with one site per unit cell

$$
H=\delta \sum_{\mathbf{j}} \sum_{n}\left|\mathbf{r}_{n}\right\rangle\left\langle\mathbf{r}_{n}+\mathbf{j}\right|,
$$

where $\mathbf{j}$ are nearest-neighbor vectors. It is readily shown that the integral formula (32) generalizes so that the ISF for the two-dimensional tight-binding system reads

$$
\frac{\int_{S} d \mathbf{k} e^{-\beta \hbar \omega(\mathbf{k})} \exp \{i t[\omega(\mathbf{k})-\omega(\mathbf{k}-\Delta \mathbf{K})]\}}{\int_{S} d \mathbf{k} e^{-\beta \hbar \omega(k)}},
$$

where $S$ is a primitive unit cell of the reciprocal lattice, and $\omega(\mathbf{k})$ is the two-dimensional dispersion relation.

When the dispersion relation is separable, the ISF factorizes into a product of two one-dimensional results. A common and relevant nonseparable example is provided by the hexagonal close-packed lattice. If the Cartesian unit vectors are $\hat{\mathbf{x}}$ and $\hat{\mathbf{y}}$, the primitive unit cell of the real-space lattice can be specified by the lattice vectors

$$
\mathbf{a}_{1}=a \hat{\mathbf{x}} ; \mathbf{a}_{2}=a[\hat{\mathbf{x}} \cos (60)+\hat{\mathbf{y}} \cos (30)] .
$$

The vectors linking nearest-neighbor sites are $\mathbf{a}_{1}, \mathbf{a}_{2}$ and $\mathbf{a}_{1}-\mathbf{a}_{2}$. The primitive unit cell of the reciprocal lattice can be specified by the reciprocal lattice vectors

$$
\mathbf{b}_{1}=b[\hat{\mathbf{x}} \cos (30)+\hat{\mathbf{y}} \cos (60)], \quad \mathbf{b}_{2}=b \hat{\mathbf{y}},
$$

where

$$
b=\frac{4 \pi}{a \sqrt{3}} .
$$

The eigenstates of the Hamiltonian are still Bloch states with crystal momentum $\mathbf{k}$ :

$$
|\mathbf{k}\rangle=\frac{1}{\sqrt{N}} \sum_{n} e^{i \mathbf{k} \cdot \mathbf{r}_{n}}\left|\mathbf{r}_{\mathbf{n}}\right\rangle,
$$

and if we write the crystal momentum in the reciprocal basis as $\mathbf{k}=\frac{1}{2 \pi}\left(k_{1} \mathbf{b}_{1}+k_{2} \mathbf{b}_{2}\right)$ then the dispersion relation can be compactly written as

$$
E(\mathbf{k})=\hbar \omega(\mathbf{k})=-\delta\left[\cos \left(k_{1}\right)+\cos \left(k_{2}\right)+\cos \left(k_{1}-k_{2}\right)\right] .
$$

The integral over a single unit cell of the reciprocal lattice can be performed independently over $0<k_{1}<2 \pi$ and $0<k_{2}<$ $2 \pi$, in other words

$$
\frac{\int_{S} d k_{1} d k_{2} e^{-\beta \hbar \omega\left(k_{1}, k_{2}\right)} \exp \left\{i t\left[\omega\left(k_{1}, k_{2}\right)-\omega\left(k_{1}-\Delta K_{1}, k_{2}-\Delta K_{2}\right)\right]\right\}}{\int_{S} d \mathbf{k} e^{-\beta \hbar \omega(k)}},
$$

However, the integral itself is nonseparable and to our knowledge cannot be expressed in closed form.

Evaluating Eq. (45) in general gives results qualitatively similar to the one-dimensional case. At diffraction conditions, where $k_{1}$ and $k_{2}$ are both multiples of $2 \pi$, the ISF is a constant. In other words all contributions to $S(\Delta \mathbf{K}, \omega)$ are elastic because $\omega(\mathbf{k})-\omega(\mathbf{k}-\Delta \mathbf{K})=0$ for all $\mathbf{k}$.

\section{B. Degenerate honeycombs}

The honeycomb system is described in real space by a unit cell as described in the previous section for a hexagonal surface, but with tight-binding sites at positions $(a / 2, c / 3)$ and $(a, 2 c / 3)$ relative to the lower left corner of each unit cell, where $c=a \cos (30)$. The tight-binding sites therefore form two interleaved sublattices. Define the nearest-neighbor jump vectors $\mathbf{j}_{1,2,3}$ as the vectors connecting a site on one sublattice to the three nearest sites on the other sublattice. Explicit expressions for $\mathbf{j}_{1,2,3}$ are given in the Appendix.

In previous sections we saw that the ISF for tunneling in a one-dimensional, one-band system and a two-dimensional, one-band system, can be expressed succinctly as $k$-space integrals. A similar general form gives the ISF for the honeycomb system, and involves both intraband and interband terms. Let $B=1,2$ be a band index, and the corresponding dispersion relations of the system be $\omega_{B}(\mathbf{k})$. Let $u_{B}(\mathbf{k})$ and $v_{B}(\mathbf{k})$ be associated functions related to the amplitudes in the eigenstates associated with the two bands. A detailed 


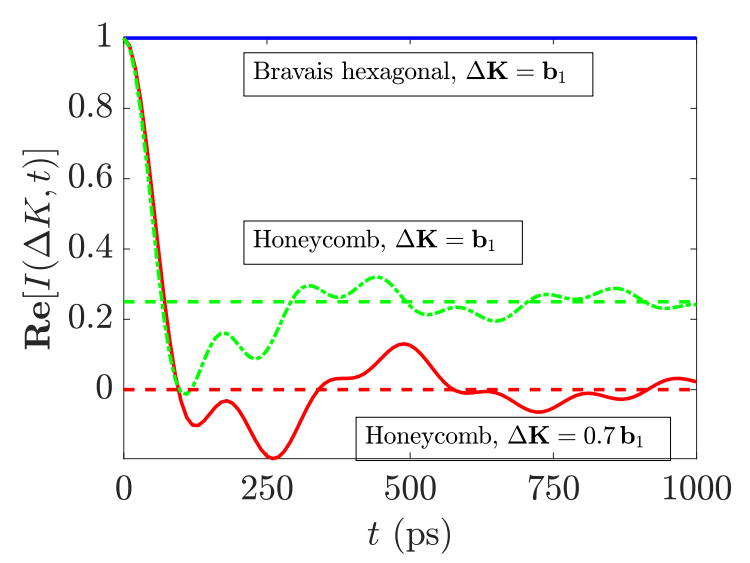

FIG. 3. The ISF $I(\Delta \mathbf{K}, t)$ for a particle coherently tunneling in a honeycomb system of degenerate tight-binding sites. The plotted curves were produced by a numerical integration of Eq. (46) at each value of the correlation time $t$. The parameters of the tight-binding system are $\delta=0.01 \mathrm{meV}, T=4.0 \mathrm{~K}$. The blue solid curve shows the trivial result at $\Delta \mathbf{K}=0$. The red solid curve shows the ISF at $\Delta \mathbf{K}=0.7 \mathbf{b}_{1}$, in other words not at any special high-symmetry condition. The green dot-dashed curve shows the ISF at $\Delta \mathbf{K}=\mathbf{b}_{1}$, and illustrates the decay to a static level greater than zero but less than unity. The static level is shown by a dashed horizontal line in each case.

derivation of $\omega_{B}, u_{B}, v_{B}$ and the construction of the ISF in those terms, is given in the Appendix. The final result is

$$
I(\Delta \mathbf{K}, t)=\frac{\int_{S} d \mathbf{k}\left|z_{B, B^{\prime}}(\mathbf{k}, \Delta \mathbf{K})\right|^{2} e^{[i t-\beta \hbar] \omega(\mathbf{k})} e^{-i t \omega(\mathbf{k}-\Delta \mathbf{K})}}{\int_{S} d \mathbf{k} \sum_{B}\left[e^{-\beta \hbar \omega_{B}(\mathbf{k})}\right]},
$$

where the complex numbers $z_{B, B^{\prime}}(\mathbf{k}, \Delta \mathbf{K})$ that are squared to give the intensities of the intraband and interband terms are given by

$$
z_{B, B^{\prime}}(\mathbf{k}, \Delta \mathbf{K})=u_{B}^{*}(\mathbf{k}) u_{B}(\mathbf{k}-\Delta \mathbf{K})+v_{B}^{*}(\mathbf{k}) v_{B}(\mathbf{k}-\Delta \mathbf{K}),
$$

and the amplitudes $u_{B}$ and $v_{B}$ are given in terms of the nearestneighbor translation vectors $\mathbf{j}_{1,2,3}$ by

$$
u_{B}(\mathbf{k})=\frac{1}{\sqrt{2}}
$$

and

$$
v_{B}(\mathbf{k})= \pm \frac{1}{\sqrt{2}} \frac{e^{-i \mathbf{k} \cdot \mathbf{j}_{1}}+e^{-i \mathbf{k} \cdot \mathbf{j}_{2}}+e^{-i \mathbf{k} \cdot \mathbf{j}_{3}}}{\mid e^{-i \mathbf{k} \cdot \mathbf{j}_{1}}+e^{-i \mathbf{k} \cdot \mathbf{j}_{2}}+e^{-i \mathbf{k} \cdot \mathbf{j}_{3} \mid}}
$$

Figure 3 shows the ISF computed by numerical integration of the expression (46). To illustrate a major difference between the Bravais-lattice hexagonal result (45) and the honeycomb result, the ISF is evaluated at zero momentum transfer, at a diffraction condition, and at an intermediate condition. At $\Delta \mathbf{K}=0$, the ISF for any system is constant in time by construction. At an intermediate condition the honeycomb ISF undergoes an oscillatory decay, qualitatively similar to the one-dimensional and Bravais-hexagonal results. At the diffraction condition $\Delta \mathbf{K}=\mathbf{b}_{1}$, the honeycomb result is not constant but decays to a static level less than unity.
However, the Bravais-hexagonal result is unity for all time due to the single-band nature of the system. The perfectly elastic ISF at diffraction conditions is familiar from jump models of surface diffusion as the minima in the Chudley-Elliott decay rate described by Eq. (35). The modification of the ISF for jump motion on non-Bravais lattice systems, which leads to nonstatic ISF at diffraction conditions, is also well known in the context of surface and bulk jump diffusion [27,69]. The result (46) encodes similar information, but for the opposite limit of perfectly coherent motion.

The finite static level can be traced back to the intraband terms, and associated with the periodicity of each individual band. In contrast, the ISF for free, ballistic quantum motion does not have a static level at any nonzero $\Delta \mathbf{K}$, which in the present language is because the free particle has an infinite number of bands within any (arbitrarily selected) first Brillouin zone, which allows the relative contribution of intraband terms to vanish.

\section{EXPERIMENTAL PROSPECTS}

The hexagonal and honeycomb examples treated above are particularly motivated by the potential application to the two-dimensional surface diffusion of hydrogen on closepacked surfaces, where adsorption sites are typically [70] the threefold hollow sites of which there are two symmetrically inequivalent types per unit cell. In classical or incoherent jump dynamics, symmetrically inequivalent threefold sites reveal themselves in the ISF via biexponential decays representing transfer of probability between different sublattices as well as among the sites on each sublattice [27,69]. Energetic inequivalence $\Delta E$ between the adsorption sites manifests itself via occupation probabilities $\propto \exp (-\beta \Delta E)$ and a corresponding detailed-balance condition on the jump rates. By comparison, the band structure of a hydrogen atom on the periodic surface exhibits an extreme sensitivity to the degree of nondegeneracy due to the strong dependence of quantum transmission amplitudes on barrier thickness [8,49]. For example, distorting a first-principles potential energy surface for $\mathrm{H} / \mathrm{Ni}$ (111) to make both threefold sites degenerate increases the lowest-energy band width by a factor of $10^{2}$ [24]. Therefore while studies of classical jump dynamics offer a method to provide strong constraints on relative adsorption energies [39,62], quantum tunneling effects could, in future, provide an even more sensitive indicator of the energetics.

The prospects for experimental observation of coherent tunneling using HeSE depend on whether long-range coherent surface diffusion could actually occur on any specific surface, and also whether the process could reasonably be observed in the experiment. The first issue is not at all straightforward to answer conclusively. It is relevant to note that no specific evidence for coherent motion of hydrogen has been derived from several previous spin echo observations of hydrogen diffusion $[24,62,71,72]$ and therefore in a serious search for coherent effects it would be beneficial to consider all the surface system properties that could promote coherence, including minimizing the coverage and temperature, and selecting or even engineering the surface to maximise the bandwidth of the lowest-energy band for hydrogen motion. The limiting factor in the realization of long-range coherent diffusion is 
likely to be the interaction with the substrate heat bath, leading to dissipation and incoherent tunneling rather than coherent propagation [42-44,65,73,74]. However, even for the twostate dissipative system under the standard simplifying model of a harmonic bath, the transition from coherent to incoherent relaxation is not straightforward, as it depends on the absolute strength of coupling to the heat bath, the $\omega \rightarrow 0$ frequency dependence of the coupling, and the temperature [22,75]. In addition to affecting the precise interplay between the system and bath dynamics, changes in temperature can also have an abrupt effect on the nature of the available substrate excitations [21], potentially leading to a drastic reduction in dissipation and dephasing effects at very low temperatures that could enable long-range coherent motion.

The correlation time plotted in Fig. 1 is routinely achievable using current helium-3 surface spin echo (HeSE) instrumentation $[20,39,62,63]$. Further, the strong off-specular helium scattering cross sections of isolated adsorbed atoms [76] allows the measurement of dilute systems as required to observe long-range independent motion of distinct adsorbates. Measurements using momentum transfers up to $\Delta K \approx 4 \AA^{-1}$ are feasible, and $a=2.7 \AA$ is a reasonable nominal value for the separation of equivalent adsorption sites on a closepacked metal substrate. The tight-binding parameter $\delta$ can be interpreted via Eq. (9) as approximately a quarter of the bandwidth of the lowest band in the dispersion relation $E(\mathbf{K})$ for surface hydrogen. The width of the lowest band for $\mathrm{H} / \mathrm{Ni}(111)$ diffusion has been computed as $5 \times 10^{-4} \mathrm{meV}$ [24], whereas the plotted value $\delta=0.01$ is a more optimistic estimate based on the hypothetical close-packed surface with degenerate fcc and hcp sites. The pessimistic bandwidth is comparable to the present energy resolution of the Cambridge HeSE instrument in the sense of complete spectral reconstruction of $S(\Delta K, \Delta \omega)$ [20,77,78], but there is ample scope for improving the energy resolution with independently proven instrumentation upgrades, for example, by increasing the maximum magnetic field integral for the ${ }^{3} \mathrm{He}$ nuclear spin manipulations [79]. Additionally, since the spin-echo method operates in a quasitime domain, full spectral reconstruction is not compulsory to interpret the partial line shape in the time domain, which leads to a substantially higher effective resolution given a suitable physical model for the ISF $[19,77,80]$. A stronger constraint might be imposed by temperature requirements. Regardless of the effect of temperature on dissipation, very low temperatures would be required to observe the imaginary component of the line shape.

\section{CONCLUSION}

The real and imaginary parts of the quantum intermediate scattering function have been derived for a particle performing long-range coherent quantum diffusion in tightbinding systems. Exact closed-form results are given for a one-dimensional system with one tight -binding site per unit cell. The derivation can be facilitated by a closed-form special case of the Baker-Campbell-Hausdorff theorem. The result has been extended to tunneling in hexagonal lattices via a k-space integral. The analogous result for tunneling in degenerate honeycomb systems is expressed in similar terms by including intraband and interband contributions to the ISF.
The relevance of the analytical result to experimental measurements of the surface diffusion of hydrogen has been discussed in the context of the current capabilities of the helium-3 surface spin echo method, and realistic theoretical band widths for hydrogen adsorbed on metal surfaces. Coherent diffusion effects are on the limit of what could be observed, in principle, given the present energy resolution of the Cambridge implementation of the spin-echo method, and could be brought firmly into the observable range by eminently feasible improvements to the instrumentation, although the associated temperature and dissipation requirements may be more difficult to realize and control.

\section{ACKNOWLEDGMENTS}

P.S.M.T. acknowledges the Engineering and Physical Sciences Research Council for doctoral funding, which enabled the core of the present work to be carried out. P.S.M.T. thanks Dr. Marco Matone for advice on the broader context of the $\mathrm{BCH}$ theorem, and the SMF Group at Cambridge for helpful discussions and support.

\section{APPENDIX: ISF IN HONEYCOMB GEOMETRY}

The honeycomb arrangement of tight-binding sites, discussed in Sec. IV B has the same unit cell as the hexagonal system discussed in Sec. IV A, but each unit cell contains two inequivalent sites. Introduce states $|\mathbf{r}\rangle$ to label localized states at the first type of site, and $|\mathbf{s}\rangle$ to label the second type. Introduce also the three vectors $\mathbf{j}_{1,2,3}$ that translate a particle on sites of type 1 to a nearest-neighbor site of type 2 . In terms of the real-space lattice vectors $\mathbf{a}_{1,2}$ defined in Sec. IV A, the nearest-neighbor translation vectors in the honeycomb system are

$$
\begin{aligned}
& \mathbf{j}_{1}=\frac{1}{3}\left(\mathbf{a}_{1}+\mathbf{a}_{2}\right), \\
& \mathbf{j}_{2}=\frac{1}{3}\left(\mathbf{a}_{2}-2 \mathbf{a}_{1}\right), \\
& \mathbf{j}_{3}=\frac{1}{3}\left(\mathbf{a}_{1}-2 \mathbf{a}_{2}\right),
\end{aligned}
$$

which, for example, can be identified with the nearestneighbor jump vectors in studies of hopping diffusion between hollow sites [27].

We define a convention for the present problem where $\mathbf{s}_{n}=\mathbf{r}_{n}+\mathbf{j}_{1}$, in other words one of the three jump vectors maps between sites in the same primitive unit cell; the other two jump vectors translate onto sites in neighboring unit cells. Then, the Hamiltonian of a nearest-neighbor, tight-binding honeycomb system can be defined by its action on each type of site

$$
\begin{aligned}
& H|\mathbf{r}\rangle=\delta\left(\left|\mathbf{r}+\mathbf{j}_{1}\right\rangle+\left|\mathbf{r}+\mathbf{j}_{2}\right\rangle+\left|\mathbf{r}+\mathbf{j}_{3}\right\rangle\right), \\
& H|\mathbf{s}\rangle=\delta\left(\left|\mathbf{s}-\mathbf{j}_{1}\right\rangle+\left|\mathbf{s}-\mathbf{j}_{2}\right\rangle+\left|\mathbf{s}-\mathbf{j}_{3}\right\rangle\right)
\end{aligned}
$$

Bloch states are still guaranteed to be eigenstates of the system, but must include amplitude on both sublattices. Let $n$ be a site index running over all sites in the system, and define 
two types of Bloch state $|a\rangle$ and $|b\rangle$ containing amplitude on one type of site only

$$
\begin{aligned}
& |a\rangle=\frac{1}{\sqrt{N}} \sum_{n} e^{i \mathbf{k r}_{n}}\left|\mathbf{r}_{n}\right\rangle, \\
& |b\rangle=\frac{1}{\sqrt{N}} \sum_{n} e^{i \mathbf{k s}_{n}}\left|\mathbf{s}_{n}\right\rangle,
\end{aligned}
$$

the action of the Hamiltonian on the states $|a\rangle$ and $|b\rangle$ can be worked out by noting that $\mathbf{r}_{n}+\mathbf{j}_{1}=\mathbf{s}_{n}, \mathbf{r}_{n}+\mathbf{j}_{2}=\mathbf{s}_{n}-\mathbf{a}_{1}$, and $\mathbf{r}_{n}+\mathbf{j}_{3}=\mathbf{s}_{n}-\mathbf{a}_{2}$, with analogous results for translating from $|\mathbf{s}\rangle$ sites to $|\mathbf{r}\rangle$ sites. Introducing an auxiliary factor

$$
A(\mathbf{k})=e^{-i \mathbf{k} \mathbf{j}_{1}}+e^{-i \mathbf{k} \mathbf{j}_{2}}+e^{-i \mathbf{k} \mathbf{j}_{3}},
$$

the results can be written as

$$
\begin{gathered}
H|a\rangle=\delta A(\mathbf{k})|b\rangle, \\
H|b\rangle=\delta A^{*}(\mathbf{k})|a\rangle .
\end{gathered}
$$

Introduce a Bloch state with a band index $B=1,2$ as a linear combination of $|a\rangle$ and $|b\rangle$ :

$$
|\mathbf{k}, B\rangle=u_{B}(\mathbf{k})|a\rangle+v_{B}(\mathbf{k})|b\rangle .
$$

The choices

$$
u_{B}(\mathbf{k})=\frac{1}{\sqrt{2}}
$$

and

$$
v_{B}(\mathbf{k})= \pm \frac{1}{\sqrt{2}} \frac{A(\mathbf{k})}{|A(\mathbf{k})|}
$$

make $|\mathbf{k}, B\rangle$ an eigenstate of $H$ with corresponding eigenvalue

$$
E(\mathbf{k})= \pm \delta|A(\mathbf{k})|,
$$

which can be verified by direct substitution. We choose $B=1$ to correspond to the positive sign in Eqs. (A13) and (A14), and $B=2$ the negative sign.

To compute the ISF we start from the dynamical structure factor (3) generalized to two spatial dimensions and including contributions from two bands

$$
S(\Delta K, \omega) \propto \sum_{\mathbf{k}, B ; \mathbf{k}^{\prime}, B^{\prime}} e^{-\beta \hbar \omega_{B^{\prime}}\left(\mathbf{k}^{\prime}\right)}\left|\left\langle\mathbf{k}, B\left|e^{i \Delta \mathbf{K} \mathbf{x}}\right| \mathbf{k}^{\prime}, B^{\prime}\right\rangle\right|^{2} \delta\left\{\omega-\left[\omega(\mathbf{k})-\omega\left(\mathbf{k}^{\prime}\right)\right]\right\}
$$

and take the Fourier transform which gives us the normalized ISF as

$$
I(\Delta K, t)=\frac{1}{Z} \sum_{\mathbf{k}, B ; \mathbf{k}^{\prime}, B^{\prime}} e^{-\beta \hbar \omega_{B^{\prime}}\left(\mathbf{k}^{\prime}\right)}\left|\left\langle\mathbf{k}, B\left|e^{i \Delta \mathbf{K x}}\right| \mathbf{k}^{\prime}, B^{\prime}\right\rangle\right|^{2} \exp \left\{i t\left[\omega(\mathbf{k})-\omega\left(\mathbf{k}^{\prime}\right)\right]\right\} .
$$

The matrix element $\left\langle\mathbf{k}, B\left|e^{i \Delta \mathbf{K} \mathbf{x}}\right| \mathbf{k}^{\prime}, B^{\prime}\right\rangle$ can be evaluated by inserting

$$
\sum_{n}\left|\mathbf{r}_{n}\right\rangle\left\langle\mathbf{r}_{n}\left|+\sum_{n}\right| \mathbf{s}_{n}\right\rangle\left\langle\mathbf{s}_{n}\right|
$$

as a resolution of the identity on either side of the operator $e^{i \Delta \mathbf{K} \mathbf{x}}$. Using $\left\langle\mathbf{r}_{n} \mid \mathbf{r}_{n^{\prime}}\right\rangle=\left\langle\mathbf{s}_{n} \mid \mathbf{s}_{n^{\prime}}\right\rangle=\delta_{n, n^{\prime}}$ and $\left\langle\mathbf{r}_{n} \mid \mathbf{s}_{n^{\prime}}\right\rangle=0$, the result simplifies to

$$
\left\langle\mathbf{k}, B\left|e^{i \Delta \mathbf{K} \mathbf{x}}\right| \mathbf{k}^{\prime}, B^{\prime}\right\rangle=\frac{1}{N} \sum_{n} u_{B}^{*}(\mathbf{k}) u_{B^{\prime}}\left(\mathbf{k}^{\prime}\right) e^{-i\left\{\mathbf{k}^{\prime}-[\mathbf{k}-\Delta \mathbf{K}]\right\} \mathbf{r}_{n}}+\frac{1}{N} \sum_{n} v_{B}^{*}(\mathbf{k}) v_{B^{\prime}}\left(\mathbf{k}^{\prime}\right) e^{-i\left\{\mathbf{k}^{\prime}-[\mathbf{k}-\Delta \mathbf{K}]\right\} \mathbf{s}_{n}}
$$

The sum $\sum_{n}$ over all unit cells generates a Kronecker- $\delta$ leading to a $k$-conservation condition. $\mathbf{k}^{\prime}=\mathbf{k}-\Delta \mathbf{K}$, and the subsequent sum over $\mathbf{k}^{\prime}$ in the ISF numerator leads to a single $\mathbf{k}$-space sum. Defining the amplitude

$$
z_{B, B^{\prime}}(\mathbf{k}, \Delta \mathbf{K})=u_{B}^{*}(\mathbf{k}) u_{B^{\prime}}(\mathbf{k}-\Delta \mathbf{K})+v_{B}^{*}(\mathbf{k}) v_{B^{\prime}}(\mathbf{k}-\Delta \mathbf{K}),
$$

the ISF can be expressed in the succinct form given in the main text

$$
I(\Delta \mathbf{K}, t)=\frac{\int_{S} d \mathbf{k}\left|z_{B, B^{\prime}}(\mathbf{k}, \Delta \mathbf{K})\right|^{2} e^{[i t-\beta \hbar] \omega(\mathbf{k})} e^{-i t \omega(\mathbf{k}-\Delta \mathbf{K})}}{\int_{S} d \mathbf{k} \sum_{B}\left[e^{-\beta \hbar \omega_{B}(\mathbf{k})}\right]},
$$

which accounts for all interband and intraband terms.

As for the hexagonal Bravais-lattice calculation (Sec. IV A), the k-space integration can be performed over any primitive reciprocal space unit cell, such that there is no redundancy in the description of the bands. Writing $\mathbf{k}$ in the reciprocal basis defined in Sec. IV A, that is $\mathbf{k}=\frac{1}{2 \pi}\left(k_{1} \mathbf{b}_{1}+k_{2} \mathbf{b}_{2}\right)$, the integrals $\int_{S} d \mathbf{k}$ can be expressed as $\int_{0}^{2 \pi} d k_{1} d k_{2}$. The k-dependent functions in the integrand can be expressed as functions of $k_{1}$ and $k_{2}$ using the expansion of the $\mathbf{j}$ vectors in the real-space lattice basis above Eq. (A1), for example,

$$
\omega(\mathbf{k})= \pm \delta\left|e^{i\left(k_{1}+k_{2}\right) / 3}+e^{i\left(k_{2}-2 k_{1}\right) / 3}+e^{i\left(k_{1}-2 k_{2}\right) / 3}\right| .
$$


[1] G. Gamow, Z. Phys. 51, 204 (1928).

[2] A. B. Balantekin and N. Takigawa, Rev. Mod. Phys. 70, 77 (1998).

[3] A. Heuer and U. Haeberlen, J. Chem. Phys. 95, 4201 (1991).

[4] S. Andersson, G. Nyman, A. Arnaldsson, U. Manthe, and H. Jónsson, J. Phys. Chem. A 113, 4468 (2009).

[5] S. Washburn, R. A. Webb, R. F. Voss, and S. M. Faris, Phys. Rev. Lett. 54, 2712 (1985).

[6] L. J. Lauhon and W. Ho, Phys. Rev. Lett. 85, 4566 (2000).

[7] A. V. Bednyakov, B. A. Kniehl, A. F. Pikelner, and O. L. Veretin, Phys. Rev. Lett. 115, 201802 (2015).

[8] L. Landau and E. Lifshitz, Quantum Mechanics (Nonrelativistic Theory), 3rd ed., Course of Theoretical Physics Vol. 3 (Butterworth-Heinemann, Oxford, 1977).

[9] A. O. Caldeira and A. J. Leggett, Ann. Phys. (NY) 149, 374 (1983).

[10] S. Coleman, Phys. Rev. D 15, 2929 (1977).

[11] A. Altland and B. Simons, Condensed Matter Field Theory (Cambridge University Press, Cambridge, England, 2007).

[12] D. Loss and D. P. DiVincenzo, Phys. Rev. A 57, 120 (1998).

[13] A. W. Chin, S. F. Huelga, and M. B. Plenio, Philos. Trans. R. Soc. London A 370, 3638 (2012).

[14] N. Lambert, Y.-N. Chen, Y.-C. Cheng, C.-M. Li, G.-Y. Chen, and F. Nori, Nat. Phys. 9, 10 (2012)..

[15] J.-D. Picon, M. N. Bussac, and L. Zuppiroli, Phys. Rev. B 75, 235106 (2007).

[16] T. Mitsui, M. Rose, E. Fomin, D. Ogletree, and M. Salmerón, Surf. Sci. 540, 5 (2003).

[17] A. Jewell, G. Peng, M. Mattera, E. Lewis, C. Murphy, G. Kyriakou, M. Mavrikakis, and E. Sykes, ACS Nano 6, 10115 (2012).

[18] F. Mezei, Z. Phys. 255, 146 (1972).

[19] G. Alexandrowicz and A. P. Jardine, J. Phys.: Condens. Matter 19, 305001 (2007).

[20] A. P. Jardine, H. Hedgeland, G. Alexandrowicz, W. Allison, and J. Ellis, Prog. Surf. Sci. 84, 323 (2009).

[21] H. Wipf, D. Steinbinder, K. Neumaier, and P. Gutsmiedl, Europhys. Lett. 4, 1379 (1987).

[22] A. Leggett, S. Chakravarty, A. Dorsey, M. Fisher, A. Garg, and W. Zwerger, Rev. Mod. Phys. 59, 1 (1987).

[23] E. Mcintosh, Adsorption and dynamics of interacting surface systems, Ph.D. thesis, University of Cambridge, Cambridge, England, 2013.

[24] J. Zhu, Study of quantum effects in adsorbate diffusion, Ph.D. thesis, University of Cambridge, Cambridge, England, 2015.

[25] J. L. Vega, R. Guantes, S. Miret-Artés, and D. A. Micha, J. Chem. Phys. 121, 8580 (2004).

[26] S. Miret-Artés and E. Pollak, J. Phys.: Condens. Matter 17, S4133 (2005).

[27] F. Tuddenham, H. Hedgeland, A. P. Jardine, B. A. J. Lechner, B. J. Hinch, and W. Allison, Surf. Sci. 604, 1459 (2010).

[28] S. Paterson, W. Allison, H. Hedgeland, J. Ellis, and A. P. Jardine, Phys. Rev. Lett. 106, 256101 (2011).

[29] I. Calvo-Almaz'an, S. Miret-Artés, and P. Fouquet, J. Phys.: Condens. Matter 24, 104007 (2012).

[30] P. S. M. Townsend and A. W. Chin, Phys. Rev. A 98, 022106 (2018).

[31] H. Hedgeland, The development of quasi-elastic helium-3 spinecho spectroscopy as a tool for the study of surface dynamics,
Ph.D. thesis, University of Cambridge, Cambridge, England, 2006.

[32] A. Alderwick, A. P. Jardine, H. Hedgeland, D. MacLaren, W. Allison, and J. Ellis, Rev. Sci. Instrum. 79, 123301 (2008).

[33] D. Chisnall, A high sensitivity detector for helium atom scattering, Ph.D. thesis, University of Cambridge, Cambridge, England, 2012.

[34] D. J. Ward, A study of spin-echo lineshapes in helium atom scattering from adsorbates, Ph.D. thesis, University of Cambridge, Cambridge, England, 2013.

[35] B. A. J. Lechner, H. Hedgeland, W. Allison, J. Ellis, and A. P. Jardine, Rev. Sci. Instrum. 84, 026105 (2013).

[36] A. Jones, A. Tamtögl, I. Calvo Almazán, and A. Hansen, Sci. Rep. 6, 27776 (2016).

[37] A. Tamtögl, B. Davey, D. J. Ward, A. P. Jardine, J. Ellis, and W. Allison, Rev. Sci. Instrum. 89, 023902 (2018).

[38] P. R. Kole, Dynamics and morphology of metal and metal oxide surfaces, Ph.D. thesis, University of Cambridge, Cambridge, England, 2011.

[39] B. A. J. Lechner, P. R. Kole, H. Hedgeland, A. P. Jardine, W. Allison, B. J. Hinch, and J. Ellis, Phys. Rev. B 89, 121405 (2014).

[40] J. Singleton, Band Theory and Electronic Properties of Solids, Oxford Master Series in Condensed Matter Physics (Oxford University Press, New York, 2001).

[41] N. W. Ashscroft and D. N. Mermin, Solid State Physics, 1st ed. (Academic, New York, 2003).

[42] U. Weiss and M. Wollensak, Phys. Rev. B 37, 2729 (1988).

[43] M. Sassetti, M. Milch, and U. Weiss, Phys. Rev. A 46, 4615 (1992).

[44] R. Egger, C. H. Mak, and U. Weiss, Phys. Rev. E 50, R655 (1994).

[45] S. Dattagupta, H. Grabert, and R. Jung, J. Phys.: Condens. Matter 1, 1405 (1989).

[46] H. Grabert, S. Linkwitz, S. Dattagupta, and U. Weiss, Europhys. Lett. 2, 631 (1986).

[47] G. H. Weiss and A. A. Maradudin, J. Math. Phys. 3, 771 (1962).

[48] R. M. Wilcox, J. Math. Phys. 8, 962 (1967).

[49] J. Sakurai and J. Napolitano, Modern Quantum Mechanics, 2nd ed. (Addison-Wesley, Boston, 2011), Chap. 3.

[50] S. Lovesey, Theory of Neutron Scattering From Condensed Matter, International Series of Monographs on Physics (No. 72), Vol. 1 (Oxford University Press, New York, 1984).

[51] R. Martínez-Casado, A. Sanz, and S. Miret-Artés, J. Chem. Phys. 129, 184704 (2008).

[52] R. Martínez-Casado, A. Sanz, J. Vega, G. Rojas-Lorenzo, and S. Miret-Artés, Chem. Phys. 370, 180 (2010).

[53] K. Riley, M. Hobson, and S. Bence, Mathematical Methods for Physics and Engineering, 3rd ed. (Cambridge University Press, Cambridge, England, 2006).

[54] A. Van-Brunt and M. Visser, J. Phys. A: Math. Theor. 48, 225207 (2015).

[55] M. Matone, J. Geom. Phys. 97, 34 (2015).

[56] M. Matone, J. High Energy Phys. 05 (2015) 113.

[57] M. Matone, Eur. Phys. J. C 76, 610 (2016).

[58] L. Van Hove, Phys. Rev. 95, 249 (1954).

[59] W. Magnus, Commun. Pure Appl. Math. 7, 649 (1954).

[60] F. Casas, A. Murua, and M. Nadinic, Comput. Phys. Commun. 183, 2386 (2012). 
[61] DLMF, NIST Digital Library of Mathematical Functions, http: //dlmf.nist.gov/, Release 1.0.19 of 2018-06-22, edited by F. W. J. Olver, A. B. Olde Daalhuis, D. W. Lozier, B. I. Schneider, R. F. Boisvert, C. W. Clark, B. R. Miller, and B. V. Saunders.

[62] E. McIntosh, K. Wikfeldt, J. Ellis, A. Michaelides, and W. Allison, J. Phys. Chem. Lett. 4, 1565 (2013).

[63] P. Rotter, B. A. J. Lechner, A. Morherr, D. Chisnall, D. J. Ward, A. P. Jardine, J. Ellis, W. Allison, B. Eckhardt, and G. Witte, Nat. Mater. 15, 397 (2016).

[64] C. Chudley and R. Elliott, Proc. Phys. Soc. London 77, 353 (1961).

[65] U. Weiss, Quantum Dissipative Systems, 4th ed., Series in Modern Condensed Matter Physics, Vol. 13 (World Scientific, Singapore, 2012).

[66] M. Topaler and N. Makri, J. Chem. Phys. 101, 7500 (1994).

[67] A. Castro Neto, F. Guinea, N. Peres, K. Novoselov, and A. Geim, Rev. Mod. Phys. 81, 109 (2009).

[68] P. S. M. Townsend, Diffusion of light adsorbates on transition metal surfaces, Ph.D. thesis, University of Cambridge, Cambridge, England, 2018.

[69] J. Rowe, K. Sköld, H. Flotow, and J. Rush, J. Phys. Chem. Solids 32, 41 (1971).

[70] L. Kristinsdóttir and E. Skúlason, Surf. Sci. 606, 1400 (2012).
[71] A. P. Jardine, E. Y. M. Lee, D. J. Ward, G. Alexandrowicz, H. Hedgeland, W. Allison, J. Ellis, and E. Pollak, Phys. Rev. Lett. 105, 136101 (2010).

[72] F. Tuddenham, Helium atom scattering as a probe of hydrogen adsorption and dynamics, Ph.D. thesis, University of Cambridge, Cambridge, England, 2011.

[73] U. Weiss and H. Grabert, Phys. Lett. A 108, 63 (1985).

[74] A. Sanz, R. Martínez-Casado, and S. Miret-Artés, Surf. Sci. 617, 229 (2013).

[75] U. Weiss, H. Grabert, and S. Linkwitz, J. Low Temp. Phys. 68, 213 (1987).

[76] D. Farías and K.-H. Rieder, Rep. Prog. Phys. 61, 1575 (1998).

[77] P. Fouquet, A. P. Jardine, S. Dworski, G. Alexandrowicz, W. Allison, and J. Ellis, Rev. Sci. Instrum. 76, 053109 (2005).

[78] B. A. J. Lechner, Studying complex surface dynamical systems using helium-3 spin-echo spectroscopy, Ph.D. thesis, University of Cambridge, Cambridge, England, 2012.

[79] W. Walter, C. Boffo, M. Borlein, T. Kozielewski, M. Monkenbusch, M. Ohl, A. Paul, B. Schrauth, G. Sikler, and C. Tiemann, IEEE Trans. Appl. Supercond. 19, 1320 (2009).

[80] G. Alexandrowicz, Helium spin echo spectroscopy: Measuring the dynamics of atoms, molecules and surfaces, Ph.D. thesis, University of Cambridge, Cambridge, England, 2005. 